\title{
Microscopic modeling of gas-surface scattering. II. Application to argon atom adsorption on a platinum (111) surface
}

\author{
A Filinov ${ }^{1,2,3}, \mathbf{M}$ Bonitz $^{1}$ and D Loffhagen ${ }^{2}$ \\ ${ }^{1}$ Institut für Theoretische Physik und Astrophysik, \\ Christian-Albrechts-Universität, Leibnizstr. 15, D-24098 Kiel, Germany \\ 2 INP Greifswald e.V., Felix-Hausdorff-Str. 2, D-17489 Greifswald, Germany \\ 3 Joint Institute for High Temperatures RAS, Izhorskaya Str. 13, 125412 \\ Moscow, Russia \\ E-mail: filinov@theo-physik.uni-kiel.de
}

\begin{abstract}
.
A new combination of first principle molecular dynamics (MD) simulations with a rate equation model presented in the preceding paper (paper I) is applied to analyze in detail the scattering of argon atoms from a platinum (111) surface. The combined model is based on a classification of all atom trajectories according to their energies into trapped, quasi-trapped and scattering states. The number of particles in each of the three classes obeys coupled rate equations. The coefficients in the rate equations are the transition probabilities between these states which are obtained from MD simulations. While these rates are generally time-dependent, after a characteristic time scale $t_{E}$ of several tens of picoseconds they become stationary allowing for a rather simple analysis. Here, we investigate this time scale by analyzing in detail the temporal evolution of the energy distribution functions of the adsorbate atoms. We separately study the energy loss distribution function of the atoms and the distribution function of in-plane and perpendicular energy components. Further, we compute the sticking probability of argon atoms as a function of incident energy, angle and lattice temperature. Our model is important for plasma-surface modeling as it allows to extend accurate simulations to longer time scales.
\end{abstract}

Keywords: plasma-surface modeling, low-temperature plasma, gas-surface interaction, adsorption and scattering of neutral particles, thermal accommodation, equilibration time, molecular dynamics, sticking probability, energy-loss distribution, argon atom, platinum surface

Submitted to: Plasma Sources Sci. Technol. 


\section{Introduction}

The recent progress in the production of micro- and nanoelectromechanical systems, aerospace engineering, vacuum technologies, and process engineering, such as gas separation membranes and heterogeneous catalysis, have revived the interest in fundamental research of rarefied gas flows and heat transport. Here, the influence of the gas-surface interactions on the momentum and energy transfer leading to the wall slip effects is of primary interest. The resulting macroscopic properties, such as the momentum/energy accommodation coefficients, can then be efficiently used as the input parameters for scattering kernels in rarefied gas flow simulations and well-defined boundary conditions.

Plasma-surface interaction is another currently actively studied field, where surface processes involving neutral atoms and molecules are of high importance. This includes the understanding of the film formation on the surface as well as feedback effects of the surface processes to the plasma composition and discharge characteristics. The questions of interest include the adsorption of gas atoms and molecules, their sticking probability, the generation of secondary particles, as well as the dependence of the adsorption on the surface temperature and the energy and impact angle of the gas particle. These properties are often known only approximately since both measurements and simulations in a plasma environment are quite complex. Although hydrodynamic modeling and kinetic simulation of plasmas have progressed remarkably during the recent decade, the interaction of the plasma with a solid surface is typically treated on a simplified level via averaged sticking and desorption rates. Here, improvements, e.g. by obtaining energy resolved desorption rates, would allow for much more accurate modeling of low-temperature plasmas. In this paper we present new results for these quantities for the test case of argon atoms impacting on a platinum surface.

In the last decades the scattering problem of rare gas atoms from metal surfaces continued to be in the focus of both theoretical [1,2 and experimental analysis 3 . A detailed overview on the recent progress in this field can be found in Refs. 44 6]. The analyses include both classical and quantum scattering regimes. In particular, it was found that some quantum features in the diffraction patterns can be resolved even for atoms as heavy as $\operatorname{Ar}[7]$. Such a situation, however, is quite unusual and most of the scattering experiments with heavy atoms are of classical nature [8 10]. But even in this case, a classical mechanics picture of the atom-surface scattering is far from being complete and further developments are of interest.

There are several main problems for a theory. The first one is an accurate inclusion of the gassurface interaction, typically reconstructed from ab initio quantum mechanical approaches such as density functional theory (DFT). A second issue concerns a correct consideration of all energy dissipation channels, like the interaction of the gas with the surface and bulk phonons, and electron-hole excitations originating from the perturbation of the electron density of the surface atoms. These major processes have to be taken into account by a theory to describe correctly the energy transfer to the surface, the angle- and energy-dependent sticking probability, the equilibration kinetics of the adsorbate localized at the surface, and the subsequent thermal desorption, which extends over a time scale ranging from few picoseconds to milliseconds. An important test for a theoretical description is certainly provided by experimental measurements performed on different physical systems and scattering conditions.

Concerning experimental studies, the dependence of the angular distribution of scattered atoms on the incident energy of the gas atoms and the surface temperature as well as the average energy loss to the surface are typically in the focus. The latter, being the most challenging to theory, is typically defined via the so-called accommodation coefficient, i.e., the ratio of the energy loss to the incident energy of the gas particles. In particular, theories, which assume parallel momentum conservation and hard sphere scattering, are not able to capture the main experimental observation that the slope of the relative final energy changes from negative to positive when increasing the incident energy of the gas particle. This behavior has been observed e.g. for the scattering of Ar atoms and diatomic molecules $\left(\mathrm{N}_{2}\right.$ and $\left.\mathrm{O}_{2}\right)$ from a $\operatorname{Ag}(111)$ surface 11 and for the scattering of Xe atoms from a $\mathrm{Pt}(111)$ surface 12 .

A real theoretical breakthrough in the understanding of experimental data has been gained by using molecular dynamics (MD) simulations. The experimental results on scattering of $\mathrm{Xe}$ on $\mathrm{Pt}(111)$ have been reproduced by Barker et al [13]. The energy loss dis- 
tribution function and its dependence on the incident energy and angle has been accurately analyzed by Lahaye et al 14] for $\operatorname{Ar}$ on $\operatorname{Ag}(111)$.

While being in good agreement with experimental data, MD simulations alone cannot always provide a deep understanding of the underlying physics. Therefore, several theoretical models of scattering have been developed in parallel. In general, they treat the interaction of the gas atoms with the surface by coupling their vertical motion to the phonon bath and use the methods of the classical statistical physics. In this framework, Brako 15 obtained an analytical expression for the energy and momentum transfer as well as for the angular distribution. However, the corrugation of the surface was neglected for reasons of simplification. As a result, Brako's theory misses the coupling between the phonons and the parallel momentum of the atom.

The first model which took this effect into account - the so-called "washboard model" - was developed by Tully [16] and was extended in a later paper by Yan et al [17. It assumes an impulsive collision of the gas atoms with the surface, but shows difficulties in reproducing the double peak structure of the angular distribution function.

The first progress in this direction was made by Pollak et al 2. They generalized Brako's formula and the "washboard model" and presented explicit results for the joint angular and final momentum distributions, the joint distributions of final scattering energy and angle as well as the interrelationship between the average energy losses and the angular scattering distribution. The application of this theory was successful in reproducing the main experimental observations. A shift of the maximum of the angular distribution to subspecular angles at low incident energies was predicted which is due to large energy losses in the horizontal direction. In contrast, a superspecular maximum in the angular distribution was reproduced at high incident energies, when the energy loss in the vertical direction dominates. The theory predicts as well that the full width at half maximum of the angular distribution varies as the square root of the temperature. It was successfully applied to explain the scattering experiments of Ar on the $\operatorname{Ag}(111)$ surface, however, only in the regime when the incident energy is sufficiently large and one can neglect the sticking probability.

The nature of sticking of heavy atoms to clean surfaces has been intensively investigated using semiclassical perturbation theory 2,1822 . Hubbard and Miller [18 calculated the sticking probability for the $\mathrm{HeW}(110)$ and $\mathrm{NeW}(110)$ systems in the regime when the energy transfer from the surface is small. Pollak [19] derived an expression for the sticking probability in the limit of weak surface corrugation, and by assuming weak coupling to the harmonic surface phonon modes. Later, an improved theory has been presented in [20], which included the second order corrections to the angular distribution of the scattered particles. In their recent work, Sahoo and Pollak 21] employed a one-dimensional generalized Langevin equation and derived an analytic expression for the temperature-dependent energy loss. A combination with the multiple collision theory of Fan and Manson 22 allowed to determine the fraction of trapped particles after subsequent collisions (bounces) with the surface. The theory has been tested by comparison with numerical simulations for the scattering of $\mathrm{Ar}$ on a $\operatorname{LiF}(100)$ surface.

In contrast to the classical theory, a quantum mechanical formalism capable of describing the scattering, trapping, sticking, and desorption for light particles, like $\mathrm{He}$ and $\mathrm{H}$, has been also extensively studied, see Refs. 23 25] and references therein.

The effect of sticking and thermalization with the surface becomes increasingly important for surfaces covered with self-assembled monolayers consisting of long-chain functionalized molecules. This type of problem has been analyzed recently by Castejón et al [26]. It was demonstrated how the efficiency of the energy exchange and the sticking probability vary with the length of the molecules in the monolayer. Longer molecules lead to an increased surface corrugation and provide an additional dissipation channel that promotes more efficient momentum and energy accommodation, and enhanced trapping.

Another aspect, which called for a systematic investigation, is the consideration of internal degrees of freedom of the scattering projectiles, such as the molecules $\mathrm{N}_{2}$ or $\mathrm{CO}_{2}$. Following the statistical description, one samples the rotational energy of molecules according to the Boltzmann distribution [27, where the thermal occupation of the excited states is defined by the quantum number of the angular momenta operator and the rotational partition function 28]. Then, the rotational energy is splitted between two rotational degrees of freedom to describe the rotation of the molecular around its symmetry axis 28. Moreover, the incident azimuth and altitude angles of the molecular bonds are randomized according to the specified incidence conditions. In this case, the scattering from the surface can bring a molecule to an excited rotational state and, thus, can constitute an additional dissipation channel 29].

While direct MD simulations provide a detailed description of the adsorbate kinetics near the surface, such simulations are very time consuming in general. In order to overcome the restriction to relatively short time scales of the order of $100 \mathrm{ps}$, we have developed 
a new approach for the modeling of the atom-surface interaction, which is based on a combination of MD simulations with a rate equation model and which has been presented in detail in paper I 30. There, we demonstrated how MD simulations can be used to reconstruct the quasi-stationary transition rates for the desorption processes on a much longer time scale. The basic assumption used was that the thermalization of the incident atoms with thermal and subthermal energy and the phonon-bath of the surface takes place on a time scale of the order of 50 to $100 \mathrm{ps}$.

In the present paper, we provide an additional confirmation of this assumption by performing a detailed analysis of the convergence of the energy distribution functions. The focus is on Ar atom scattering from a $\mathrm{Pt}(111)$ surface, which represents a quite well studied system. Mullins et al 31] have conducted detailed experimental studies on such system and reported on the changing role of the parallel momentum, $p^{\|}$, with surface temperature, $T_{s}$, for the trapping probability. The degree to which the parallel momentum is involved in the trapping process is frequently expressed by an energy scaling factor, $\cos ^{n} \theta$, where $\theta$ is the angle of incidence of the gas atoms impinging onto the surface. The "total" energy scaling corresponds to $n=0$, while the "normal" scaling corresponds to $n=2$. The normal scaling implies that the trapping probability is a function of the normal momentum, $p^{\perp}$, only. However, the experiment showed a gradual change of the scaling factor with increasing surface temperature from $n=$ 1.5 at $T_{s}=80 \mathrm{~K}$ to $n=0.5$ at $T_{s}=300 \mathrm{~K}$. It was suggested that high surface temperatures increase the surface roughness and that parallel momentum dissipation also becomes increasingly more important to the trapping dynamics as $T_{s}$ increases.

Such deviations from the normal energy scaling have been intensively investigated by means of numerical simulation 32 36]. Both low and high temperature regimes have been analyzed. It was found that $\left\langle p^{\|}\right\rangle(t)$ shows a much slower convergence with time in comparison with $\left\langle p^{\perp}\right\rangle(t)$. This fact points to an incomplete equilibration of the parallel velocity prior to desorption. The desorbed atoms retain a memory of their incidence conditions, and this information enters into the desorption probability. Correlations with initial conditions retained during the dynamics of adatoms across the surface can be studied in depth in the simulations by monitoring the time dependence of the energy and other quantities. This approach is followed in the present studies as well. In particular, we look in detail how the energy exchange (or the energyloss function) depends on the incidence conditions: the initial momentum, energy and lattice temperature. The main non-adiabatic process is the exchange with lattice phonons.

The present work extends the earlier analyses 32 38 on the scattering of $\mathrm{Ar}$ on $\mathrm{Pt}(111)$ surfaces by including the effects of multiple reflections. This allows to resolve characteristic times for the convergence of the energy and energy-loss distribution function to a quasi-stationary state.

The paper is structured as follows. In section 2 we analyze the temporal evolution of the energy distribution of the adsorbate. The relaxation of the surface-normal and tangential energy components is analyzed in section 3 . Section 4 presents results for the sticking coefficient of argon atoms on Pt(111) surfaces. It includes an analysis of its dependence on the incident energy and angle, and on the lattice temperature as well as a comparison with experimental data available from literature. Finally, a discussion and conclusions are given in section 5 .

\section{Evolution of the energy distribution of trapped and scattered adsorbate atoms}

In this section we analyze in detail how the system evolves towards equilibrium by analyzing the convergence to an equilibrium energy distribution function, and reveal possible dependencies on the incident angle $(\theta)$, incident energy $\left(E_{i}\right)$ and lattice temperature $\left(T_{s}\right)$. We study mono-energetic gas atoms that are introduced at a height $z$ of $20 \AA$ above the surface and are incident on a surface with $\theta\left[^{\circ}\right]=0(30,60)$ and $E_{i} /\left(k_{B} T_{r} / e_{0}\right)=0.5(0.62,1.41)$ with $k_{B} T_{r} / e_{0}=25.7 \mathrm{meV}$ corresponding to the room temperature $T_{r}=300 \mathrm{~K}$. Here, the angle $\theta=0^{\circ}$ denotes normal incidence. The energies are chosen to maintain a similar value of the initial sticking probability in every case.

Argon atoms are trapped near the surface within the distance $z^{c}=r_{\mathrm{Ar}-\mathrm{Pt}}^{c} \leq 10 \AA$, where $r_{\mathrm{Ar}-\mathrm{Pt}}^{c}$ is the cut-off radius of the potential [30]. Their trajectories are followed up to $n_{b}=40$ bounces or until the atoms are scattered into the continuum, i.e., an atom can freely leave the surface region for heights $z(t)>z^{c}$. For each set of incidence conditions $\left\{\theta, E_{i}, T_{s}\right\}$, the measured distribution functions, their mean values and variances are evaluated for a statistical ensemble containing 1000 - 5000 trajectories.

When a gas atom interacts with the surface for the first time, the exchanged energy depends crucially on the incident energy, but only to a lesser extent on the lattice temperature $T_{s}$. This was confirmed by previous studies of Smith et al 34], where it was shown that the mean energy loss is insensitive to $T_{s}$, while the width of the energy-loss distribution function increases with $T_{s}$. These observations are re-examined below with full temporal resolution to identify the presence of a quasi- 

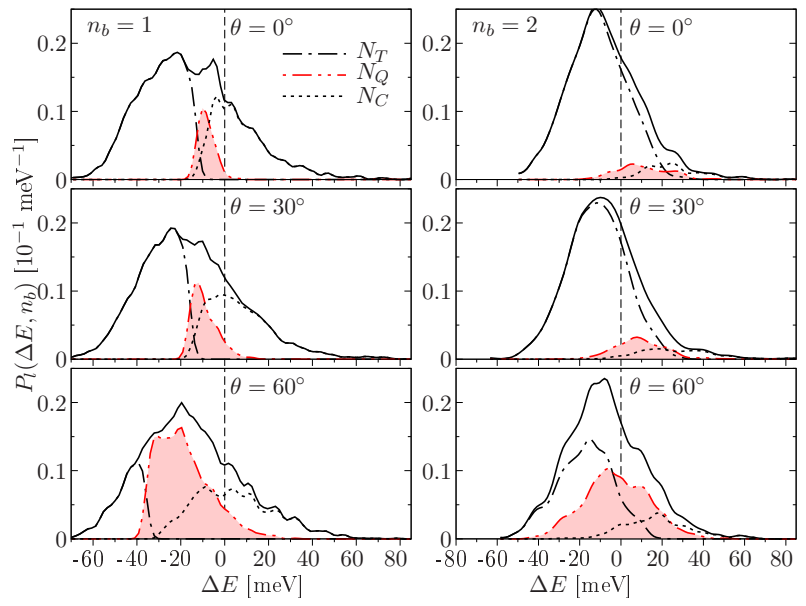

Figure 1. Energy loss distribution $P_{l}\left(\Delta E ; n_{b}\right)$ with $\Delta E=$ $E_{f}-E_{i}$ after $n_{b}=1$ (left column) and $n_{b}=2$ (right column) bounces at the lattice temperature $T_{s}=190 \mathrm{~K}$. The contribution of the trapped, quasi-trapped (filled area) and continuum states is indicated for comparison. Incident angles are $\theta=0^{\circ}\left(30^{\circ}, 60^{\circ}\right)$ and the corresponding incident kinetic energies are chosen as $E_{i} /\left(k_{B} T_{r} / e_{0}\right)=0.5(0.62,1.41)$ with $T_{r}=300 \mathrm{~K}$ corresponding to $k_{B} T_{r} / e_{0}=25.7 \mathrm{meV}$.

equilibrium phase and to justify the introduction of the equilibration time $t^{E}$ and the equilibrium transition rates $T_{\alpha \beta}^{E}$ according to paper I 30 .

\subsection{Dynamics of the energy loss distribution function}

We start our discussion with the energy loss distribution function (ELDF), which is analyzed after a different number of bounces $n_{b}$ of argon atoms with the $\mathrm{Pt}(111)$ surface. Figure 1 compares the results for the three incident angles $\theta=0^{\circ}, 30^{\circ}$, and $60^{\circ}$ at $T_{s}=190 \mathrm{~K}$ after one and two bounces. Here, $\Delta E=E_{f}-E_{i}$ is the energy change during a single bounce event with the final $\left(E_{f}\right)$ and incident $\left(E_{i}\right)$ energy, $\theta=0^{\circ}$ corresponds to normal incidence, and $N_{\mathrm{T}}$, $N_{\mathrm{Q}}$ and $N_{\mathrm{C}}$ denote the fraction of atoms in trapped $(\mathrm{T})$, quasi-trapped $(\mathrm{Q})$ and continuum $(\mathrm{C})$ states, respectively. The energy losses due to electronic friction are of minor importance in the present analysis, since the vibrational frequencies of Ar on the surface are much lower than the Debye frequency of the platinum surface. Therefore, the coupling to the high frequency electronic motion should be negligible. We have tested this by explicit inclusion of a spatially varying electronic friction term in the equations of motion and found that the corresponding energy losses are below few meV. Notice that this effect becomes important in the high-energy regime with incident energies exceeding $0.5 \mathrm{eV}$.

Figure11illustrates that the ELDF always extends from approximately -70 to $60 \mathrm{meV}$ at the conditions considered. It has a similar shape in all three cases for $n_{b}=1$ and 2 , respectively. The corresponding

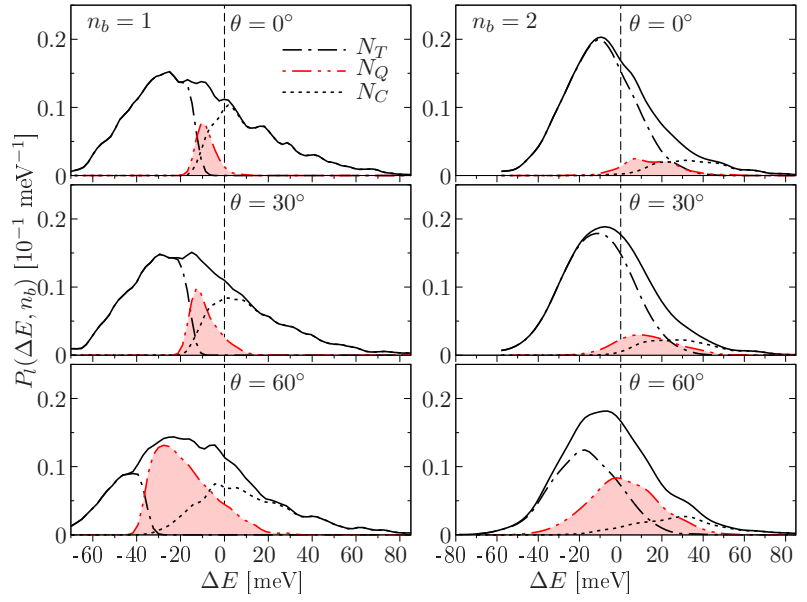

Figure 2. Same as in figure 1 but for the lattice temperature $T_{s}=300 \mathrm{~K}$.

mean value of the distributions is close to $-20 \mathrm{meV}$ for $n_{b}=1$ and $-10 \mathrm{meV}$ for $n_{b}=2$, and it does not depend on the incident angle $\theta$. However, the relative contributions of the different states, i.e., the $\mathrm{T}, \mathrm{Q}$, and $\mathrm{C}$ states, is very different. For angles around $\theta=60^{\circ}$, a large fraction of initial energy is accumulated in the parallel kinetic energy $E_{i}^{\|}=E_{i} \sin \theta$. Hence, in order to bring a particle to a trapped state, i.e., a final state with $E_{f}=E_{f}^{\perp}+E_{f}^{\|}+V<0$, the parallel momentum should be significantly perturbed by the lattice: $\Delta E \propto$ $\left(E_{f}^{\|}-E_{i}^{\|}\right) \propto-E_{i}^{\|}$. Here, $E_{f}^{\perp}, E_{f}^{\|}$, and $V$ denote the perpendicular and parallel final kinetic energy and the trapping potential of the surface, respectively,

We also observe that the value $\left(E_{f}^{\perp}+V\right)$ is similar in the three cases after the first reflection due to a similar value $E_{i}^{\perp}=E_{i} \cos \theta$ of the incident gas atoms. Hence, the energy loss of the trapped states increases with growing parallel incident kinetic energy $E_{i}^{\|}$. This explains the shift of the maximum in the ELDF of the trapped states to negative energies with increasing $\theta$. As a result, the relative contribution of the $\mathrm{T}$ states in the full distribution function is reduced and taken over by the $\mathrm{Q}$ states. This trend can be directly followed on the left side of figure 1. where the Q states are shown by the filled pattern.

The right column of figure 1 shows the ELDF after the second bounce. The contribution of the $\mathrm{T}$ states dominates for small incident angles $\left(\theta=0^{\circ}, 30^{\circ}\right)$. This results from the large transition rate $T_{T Q}$ leading to a fast conversion from $\mathrm{Q}$ to $\mathrm{T}$ states (cf. figure 9 in paper I [30]). For larger incident angles $\left(\theta=60^{\circ}\right)$, the population of the $\mathrm{Q}$ state becomes comparable to that of the $\mathrm{T}$ state for $n_{b}=2$ due to the large fraction of $N_{\mathrm{Q}}$ for $n_{b}=1$ being reduced by the second bounce.

From this first analysis, we can expect that the convergence to the quasi-equilibrium, i.e., when the $\mathrm{T}$ states dominate, takes longer for larger incident 

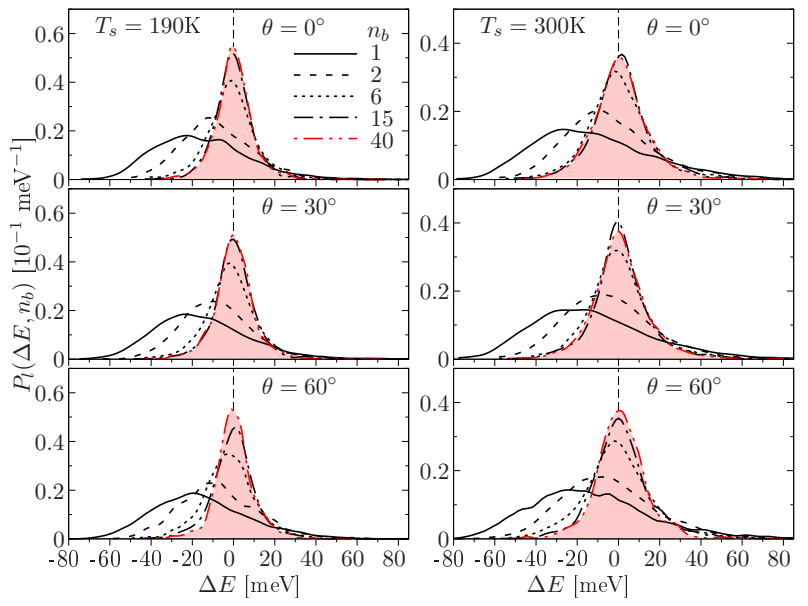

Figure 3. Energy loss distribution $P_{l}\left(\Delta E ; n_{b}\right)$ for different number $n_{b}$ of bounces with $1 \leq n_{b} \leq 40$ at different incident angles $\theta$ and $T_{s}=190 \mathrm{~K}$ (left) and $T_{s}=300 \mathrm{~K}$ (right). The converged distribution $\left(n_{b}=40\right)$ is shown by the filled pattern.

angles, because more bounces are required to reach such condition. In order to have a similar initial sticking coefficient, the energy $E_{i}$ in the incident beam has to be increased with increasing angle of incidence $\theta$ due to the $E_{i}^{\|}$component. This in turn increases the initial population of the quasi-trapped states and leads to a slower convergence to a quasi-stationary distribution.

The corresponding ELDF obtained for the larger lattice temperature $T_{s}=300 \mathrm{~K}$ is represented in figure 2. The interpretation of these results is similar as for $T_{s}=190 \mathrm{~K}$ (figure 1 ). The main difference is the thermal broadening of the ELDF occurring at all angles.

Figure 3 shows the ELDF for different number of bounces $n_{b}$ at the incident angles $\theta=0^{\circ}, 30^{\circ}$, and $60^{\circ}$ and $T_{s}=190$ and $300 \mathrm{~K}$. Because more bounces require longer time scales, this figure represents the temporal evolution of the ELDF, where the correlation between average number of bounces and the absolute time scale is displayed in figure 7 of paper I [30]. The results shown in figure 3 clearly demonstrate that the adsorbate atoms actually equilibrate. The converged distribution is always reached after $n_{b}=40$ bounces. It has a symmetric Gaussian-like form with respect to the absolute value of $\Delta E$. Here, the probabilities of excitation and de-excitation due to an absorption or emission of lattice phonons become equal.

The rate of convergence of the ELDF to the stationary form depends on the incident angle. The distribution is close to the converged result after about 15 bounces at both lattice temperatures for $\theta=0^{\circ}$ and $30^{\circ}$. In contrast, there are still some deviations from the stationary distribution for $\theta=60^{\circ}$, even for $n_{b}=15$. Here, full convergence is reached only for $n_{b} \geq 30$. The slower convergence at larger $\theta$ is directly
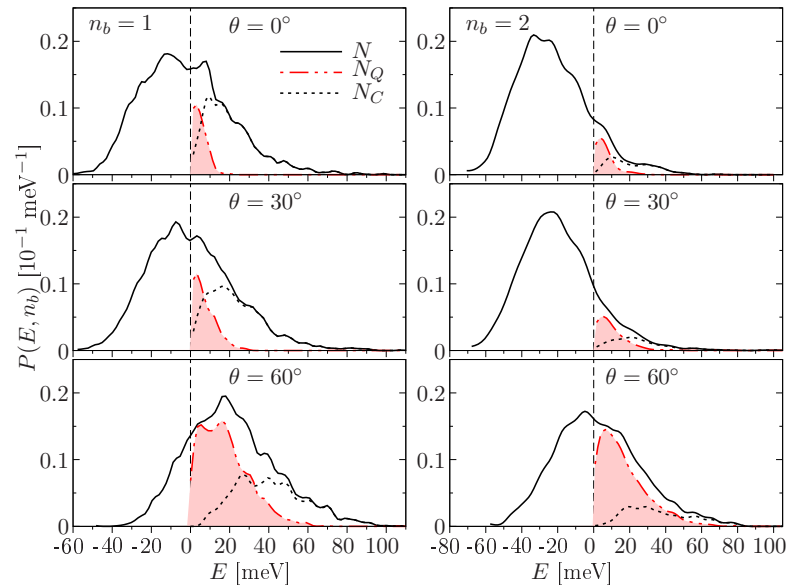

Figure 4. Total energy distribution $P\left(E, n_{b}\right)$ after $n_{b}=1$ (left column) and $n_{b}=2$ (right column) bounces at $T_{s}=190 \mathrm{~K}$ and incidence conditions as in figure 1 The contribution of the trapped states corresponds to $E<0$. The quasi-trapped $\left(N_{Q}\right)$ and continuum $\left(N_{C}\right)$ states contribute for $E \geq 0$.

related to the non-negligible contribution of the quasitrapped states.

The comparison of the results for the two lattice temperatures clearly shows that the effect of thermal broadening becomes larger with increasing $T_{s}$. A corresponding quantitative analysis is presented in table 1, where the variance $\sigma_{l}\left(\theta, T_{s}\right)$ of the ELDF is given for the three incident angles and three lattice temperatures. The variance is obtained by fitting the ELDF for $n_{b}=40$ to the Gaussian form $e^{-(\Delta E)^{2} /\left(2 \sigma_{l}^{2}\right)}$. It exhibits a linear scaling with $T_{s}$ and shows no noticeable dependence on $\theta$. This justifies that the fitted distributions have a quasi-stationary form defined purely by the lattice properties, whereas any correlations with the incidence conditions $\left(\theta, E_{i}\right)$ are practically lost.

Table 1. Variance $\sigma_{l}\left(\theta, T_{s}\right)[\mathrm{meV}]$ of the ELDF for different incident angles $\theta$ and lattice temperatures $T_{s}$. For $\theta=0^{\circ}$, $30^{\circ}$, and $60^{\circ}$ the incident gas atoms have the initial energy $E_{i} /\left(k_{B} T_{r} / e_{0}\right)=0.5,0.62$, and 1.41 , respectively.

\begin{tabular}{cccc}
\hline$T_{s}(\mathrm{~K})$ & $\theta=0^{\circ}$ & $\theta=30^{\circ}$ & $\theta=60^{\circ}$ \\
\hline 80 & $3.50(4)$ & $3.48(4)$ & $3.60(6)$ \\
190 & $7.20(10)$ & $7.48(12)$ & $7.22(10)$ \\
300 & $10.13(24)$ & $9.83(19)$ & $9.77(14)$ \\
\hline
\end{tabular}

\subsection{Dynamics of the total energy distribution function of the adsorbate atoms}

The bounces lead to a change of the total energy distribution function (EDF) of the adsorbate atoms $P\left(E, n_{b}\right)$, which is represented in the figures 4 and 5. Figure 4 presents the EDF of the adsorbate atoms after one and two bounces at the lattice temperature of $190 \mathrm{~K}$, which corresponds to the ELDF displayed 

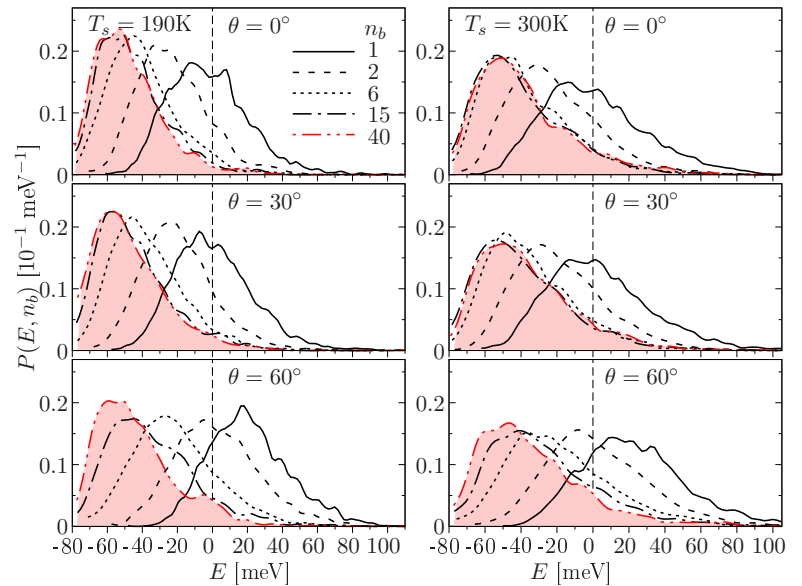

Figure 5. Total energy distribution $P\left(E, n_{b}\right)$ for different number $n_{b}$ of bounces with $1 \leq n_{b} \leq 40$ at different incident angles $\theta$ and $T_{s}=190 \mathrm{~K}$ (left) and $T_{s}=300 \mathrm{~K}$ (right). The converged distribution $\left(n_{b}=40\right)$ is shown by the filled pattern.

in figure 1. The EDF is composed of trapped, quasitrapped and continuum states, where the contribution of the $\mathrm{T}$ states corresponds to energies $E<0$ and the $\mathrm{Q}$ and $\mathrm{C}$ states contribute to the EDF for $E \geq 0$.

Figure 5 displays the EDF for different number of bounces $n_{b} \leq 40$ at $T_{s}=190$ and $300 \mathrm{~K}$, corresponding to the ELDF in figure 3 and covering the entire time scale of the MD simulations. As before, we clearly observe that the convergence to the stationary distribution depends on the incident angle and that it should be explained by the initial population of the quasi-trapped states. We also find that the converged distribution corresponds mainly to the trapped states with the negative energies comparable to the depth of the physisorption well $\left(E_{0} \approx-78 \mathrm{meV}\right)$.

Next, we analyze the evolution of the high-energy tail of the distribution functions. The tail with $E \geq 0$ is due to the quasi-trapped and continuum states. The fraction of these states typically decreases with increasing $n_{b}$ (or time). The lower or higher energies are mainly due to the $\mathrm{Q}$ and $\mathrm{C}$ states, respectively. Note that the contribution of the $\mathrm{Q}$ states vanishes above some characteristic energy $E_{c}$, which is related to the lattice temperature according to $E_{c}=\nu \cdot k_{B} T_{s}$ with $\nu=2 \ldots 3$. This effect can be clearly seen in figure 5 . For instance, the high-energy tail of the EDF at $n_{b}=40$ strongly decays above $40 \mathrm{meV}$ for $T_{s}=$ $190 \mathrm{~K}$ corresponding to $16.4 \mathrm{meV}$, and it extends up to 60 to $70 \mathrm{meV}$ at the higher temperature $T_{s}=300 \mathrm{~K}$, which corresponds to $25.7 \mathrm{meV}$.

The reason, why the contribution of the $\mathrm{Q}$ states can be observed in the EDF even in the regime of quasiequilibrium $\left(n_{b} \gtrsim 30\right)$, is directly related to the detailed balance condition derived in paper I 30

$N_{T}(t) T_{Q T}(t) \approx N_{Q}(t)\left[T_{C Q}(t)+T_{T Q}(t)\right]$,

which holds between the $\mathrm{T}$ and $\mathrm{Q}$ states. Here, $T_{Q T}$,
$T_{C Q}$, and $T_{T Q}$ denote the transition rates from $\mathrm{T}$ to $\mathrm{Q}$, from $\mathrm{Q}$ to $\mathrm{C}$, and from $\mathrm{Q}$ to $\mathrm{T}$ states, respectively. A finite but relatively small fraction of the $\mathrm{Q}$ states is sustained by the excitation channel $\mathrm{T} \rightarrow \mathrm{Q}$. By analyzing our MD data we found that this situation is common for high lattice temperatures $\left(T_{s}=190\right.$ and $300 \mathrm{~K})$, see $N_{Q}(t)$ in figures 5 and 7 of paper I 30 . The same effect is also present for the lower $T_{s}=80 \mathrm{~K}$ when the thermal desorption is strongly suppressed. It can be resolved from the curve $N_{Q}(t)$ in figure 3 of paper I when plotted in a logarithmic scale.

This behavior can be understood from the following arguments. If the desorption of the trapped states to the continuum is most probable via a twostage excitation, i.e., $\mathrm{T} \rightarrow \mathrm{Q} \rightarrow \mathrm{C}$, the quasi-trapped states should be present as a key ingredient of the desorption kinetics. In this case the high-energy tail observed in $P(E)$ is an intrinsic feature of the present system. This behavior is further supported by the temperature dependence of the transition rate $T_{Q T}^{E}$ that is analyzed in paper I.

\section{Kinetic energy distribution of adsorbate atoms: accommodation of normal and tangential energy components}

In paper I 30 two types of states temporarily localized near the surface have been introduced. Typically, the quasi-trapped trajectories are characterized by a fast accommodation of the normal velocity component 32 , 34. The equilibration times can be analyzed via the bounce number-dependent distribution functions for the parallel and normal kinetic energy components: $P\left(E^{\perp}, n_{b}\right)$ and $P\left(E^{\|}, n_{b}\right)$. They can be directly related to the time-dependent distributions using the scaling factor $c$ from the time dependence of the average bounce number, $\left\langle n_{b}\right\rangle(t) \approx c \cdot t$ (see paper I $\left.[30]\right)$. In the following, we use the value $t_{0}=10^{3} \cdot a_{0} \sqrt{m_{\mathrm{Ar}} / E_{\mathrm{h}}}=$ $6.53 \mathrm{ps}$ as a time unit, where $a_{0}$ is the Bohr radius, $m_{\text {Ar }}$ denotes the atomic mass of the argon atoms, and $E_{\mathrm{h}}=27.211 \mathrm{eV}$ is the Hartree energy.

\subsection{Distribution function $P\left(E_{k}^{\perp}, n_{b}\right)$ of the normal energy component}

First, we demonstrate the convergence of the normal component $E_{k}^{\perp}$ of the kinetic energy with increasing number of bounces $n_{b}$ in figure 6. Three incident angles $\theta$ and two lattice temperatures are compared. The incident kinetic energies are the same as in figure 1. They are visible in the initial distribution $P\left(E_{k}^{\perp}, 0.4 t_{0}\right)$, represented by the dashed blue lines with two peaks at $E_{k}^{\perp}=60$ and $80 \mathrm{meV}$, which are computed at the time $t \sim 0.4 t_{0}=2.6 \mathrm{ps}$, just before the full energy exhibits the first jump due to the inelastic scattering. Note that the value $E_{k}^{\perp}$ is shifted from 

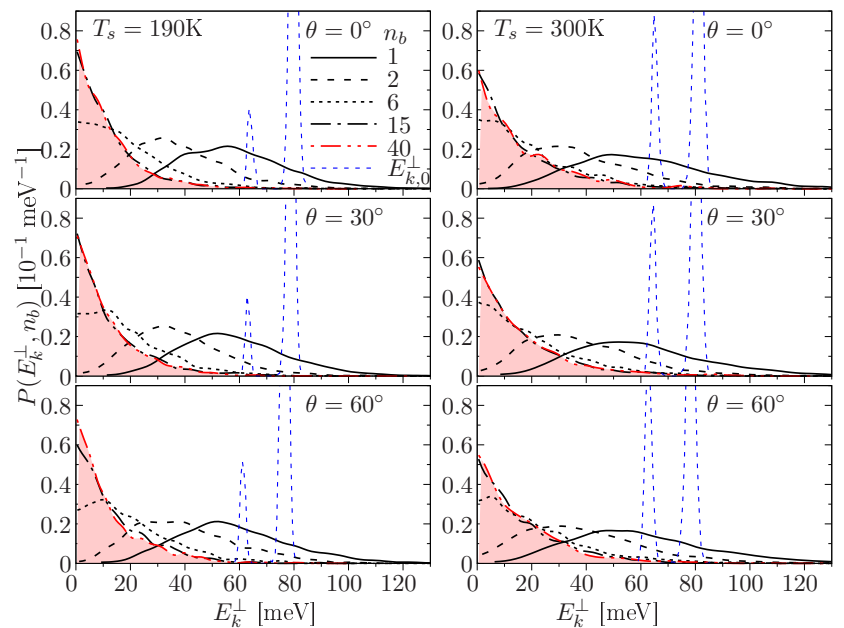

Figure 6. Distribution of the normal kinetic energy component $P\left(E_{k}^{\perp}, n_{b}\right)$ for different bounce numbers $n_{b}$ with $1 \leq n_{b} \leq 40$. Two lattice temperatures $\left(T_{s}=190\right.$ and $\left.300 \mathrm{~K}\right)$ and three incident angles $\left(\theta=0^{\circ}, 30^{\circ}, 60^{\circ}\right)$ are compared. The incident kinetic energy for each $\theta$ is specified in figure 1 The converged distribution $\left(n_{b}=40\right)$ is shown by the filled pattern, and the dashed blue lines with the two sharp peaks represent the initial distribution just before the first bounce.

the incident kinetic energy $E_{i}^{\perp}$ by the depth of the physisorption well. The particles reside at a distance of about $3-4 \AA$ from the surface and, hence, are strongly accelerated in the physisorption potential well. The initial distribution is very similar for the three angles. The two peaks correspond to the energy in the physisorption well at the two turning points, which corresponds to the atop and hollow site, respectively. This fact explains the separation of about $12 \mathrm{meV}$ between the peaks. We conclude that the initial scattering conditions are very similar for all three cases. As a result, the subsequent evolution of the distribution function $P\left(E_{k}^{\perp}, n_{b}\right)$ with increasing $n_{b}$ follows the same trend and rapidly converges for $n_{b}>6$. In particular, the distribution at $n_{b}=15$ practically coincides with the converged distribution (cf. the filled pattern, which refers to $n_{b}=40$ bounces).

The left and right panels demonstrate the effect of thermal broadening specific to the given lattice temperature. For $T_{s}=300 \mathrm{~K}$ there is a reduction of the height of the distribution function at the origin, $\left.P\left(E_{k}^{\perp}=0, n_{b}\right)\right|_{n_{b} \geq 15}$, an increased half-width and a broader high-energy tail. In conclusion, the MD simulations prove indeed a relatively fast convergence of the normal kinetic energy distribution function.

\subsection{Distribution function $P\left(E_{k}^{\|}, n_{b}\right)$ of the in-plane energy component}

A similar analysis is performed for the parallel component $E_{k}^{\|}$of the kinetic energy. The corresponding result is displayed in figure 7. It is immediately evident that
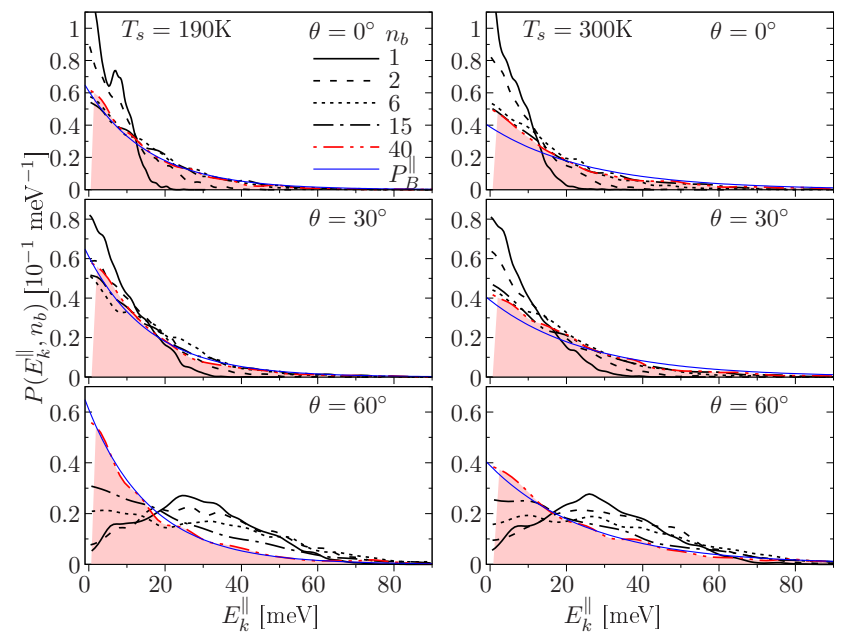

Figure 7. Distribution of the parallel kinetic energy component, $P\left(E_{k}^{\|}, n_{b}\right)$ for different bounce numbers $n_{b}$ with $1 \leq n_{b} \leq 40$. Two lattice temperatures $\left(T_{s}=190\right.$ and $\left.300 \mathrm{~K}\right)$ and three incident angles $\left(\theta=0^{\circ}, 30^{\circ}, 60^{\circ}\right)$ are compared. The converged distribution $\left(n_{b}=40\right)$ is shown by the filled pattern. The blue solid line is the prediction by the Boltzmann distribution, $P_{B}\left(E_{k}^{\|}, T_{s}\right)$.

the initial distribution function $P\left(E_{k}^{\|}, t\right)$ taken just before the first bounce at $t \sim 0.4 t_{0}$ significantly differs from the normal energy distribution shown in figure 6 . It is peaked at the parallel kinetic energy in the incident beam, $E_{i}^{\|}=E_{i} \sin ^{2} \theta$. Up to some separation distance, this value is not influenced by the gas-surface interaction and, hence, the acceleration in the physisorption potential is irrelevant. As a result, a strong correlation with the initial energy is preserved for several first bounce events.

For the three angles presented in figure 7, the parallel components have been chosen accordingly as $E_{i}^{\|}=0 \mathrm{meV}\left(\theta=0^{\circ}\right), E_{i}^{\|}=3.98 \mathrm{meV}\left(\theta=30^{\circ}\right)$, and $E_{i}^{\|}=27.26 \mathrm{meV}\left(\theta=60^{\circ}\right)$. The requirement of a similar initial sticking probability for the cases compared in section 4 results in a relatively large parallel component for $\theta=60^{\circ}$, whereas the normal components for the three cases stay comparable: $E_{i}^{\perp}=$ $12.85 \mathrm{meV}\left(\theta=0^{\circ}\right), E_{i}^{\perp}=11.95 \mathrm{meV}\left(\theta=30^{\circ}\right)$, and $E_{i}^{\perp}=9.07 \mathrm{meV}\left(\theta=60^{\circ}\right)$.

This choice of initial parameters explains to a large extent the slope of the distribution function after the first bounce. Due to a broad energy perturbation, $\Delta E \sim 15 \mathrm{meV}$, in a single inelastic scattering event, specified e.g. by the half-width of the distribution for $\theta=0^{\circ}$ at $n_{b}=1$, no qualitative difference is observed for the incident angles $0^{\circ}$ and $30^{\circ}$ when $E_{i}^{\|} \ll \Delta E$. For $\theta=60^{\circ}$ we observe a broad distribution of similar halfwidth $\Delta E$, centered around the initial energy $E_{i}^{\|} \sim$ $27 \mathrm{meV}$. It gradually converges to the filled pattern $\left(n_{b}=40\right)$ with the increase of the bounce number, but the convergence is much slower than for the normal 
component $E^{\perp}$ (cf. figure 6). Even for $n_{b}=15$ we still observe from figure 7 that the population at low energies is reduced at the expense of the population at high energies. The distribution above $E_{i}^{\|}$starts to decay significantly only for $6 \leq n_{b} \leq 15$ or within $10 \mathrm{ps} \leq t \leq 20 \mathrm{ps}$. On the same time scale, the fraction of the quasi-trapped states $N_{Q}(t)$ is reduced from $20 \%$ to $8 \%$. Hence, there is a direct relation between the $N_{Q}$ fraction and the population of high-energy states in $P\left(E_{k}^{\|}, n_{b}\right)$. The faster the depletion of $N_{Q}(t)$ due to the decay channels $\mathrm{Q} \rightarrow \mathrm{T}$ and $\mathrm{Q} \rightarrow \mathrm{C}$ takes place, the faster the convergence of the in-plane EDF to the quasi-equilibrium form is. The value of the transition rates $T_{C Q(T Q)}$ (see paper I 30$]$ ), in its turn, crucially depends on the surface kinetics and the smoothness of the potential energy surface (PES) reconstructed by the Ar-Pt(111) interaction potential. For a flat PES the parallel kinetic energy component is less perturbed during a bounce event, and the conversion of the EDF to its stationary form takes longer.

As an example, we consider the small incident angles $\theta=0^{\circ}$ and $30^{\circ}$. Here, the $N_{Q}$ fraction is small already after the first bounce (see figure 5 of paper I). As a result, at $t=20 \mathrm{ps}$ (or $\left\langle n_{b}\right\rangle \sim 15$ ) its relative contribution to $P\left(E_{k}^{\|}, n_{b}\right)$ is below $3 \%$ in figure 7 , and the trapped states dominate. We can observe that the distribution function (DF) is close to its stationary form already after few bounces $\left(n_{b} \sim 6\right)$ in this case.

Now the question arises why the distribution $P\left(E_{k}^{\perp}, n_{b}\right)$ converges much faster than $P\left(E_{k}^{\|}, n_{b}\right)$. The comparison of figures 6 and 7 makes clear that $P\left(E_{k}^{\perp}, n_{b}\right)$ contains the same amount of the quasitrapped states as $P\left(E_{k}^{\|}, n_{b}\right)$ in figure 7. However, its transformation to the stationary form is reached already after few bounces $\left(n_{b} \sim 10\right)$. Moreover, the initial DF is peaked at a much higher energy $(60-$ $80 \mathrm{meV}$ ). One main reason is the trapping condition $E_{k}^{\perp}+E_{p}<0$ with the potential energy $E_{p}$, which specifies an upper bound for the possible values of $E_{k}^{\perp}$. Hence, all trajectories with the high-energy normal component vanish to the scattered fraction very fast.

\subsection{Relaxation of the in-plane EDF at low temperature}

In figure 8 we analyze the convergence behavior of the distribution of the parallel kinetic energy component at low lattice temperature $\left(T_{s}=80 \mathrm{~K}\right)$ and two incident angles $\left(30^{\circ}, 60^{\circ}\right)$. In contrast to the higher temperatures (cf. figure 7), the convergence is not achieved at $n_{b}=15$. There are significant deviations from the DF at $n_{b}=40$. They can be better followed if the DF is compared with the Boltzmann prediction for the lattice temperature of $80 \mathrm{~K}$, see Eq. (2) below. Indeed, both distributions seem to converge to the
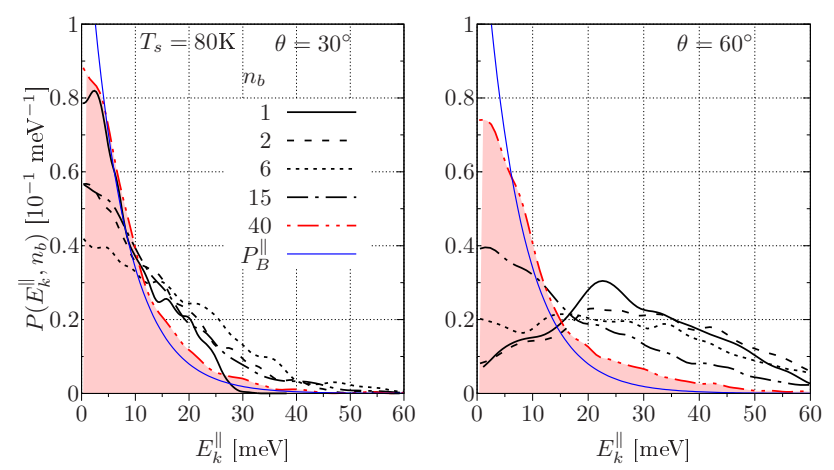

Figure 8. Distribution of the parallel kinetic energy component, $P\left(E_{k}^{\|} ; n_{b}\right)$, at low lattice temperature, $T_{s}=80 \mathrm{~K}$, at different bounce numbers $1 \leq n_{b} \leq 40$. Two incident angles $(\theta=$ $\left.30^{\circ}, 60^{\circ}\right)$ are compared. The converged distribution $\left(n_{b}=40\right)$ is shown by the filled pattern. The blue solid line is the prediction by the Boltzmann distribution, $P_{B}\left(E_{k}^{\|}, T_{s}\right)$.

thermodynamic prediction, when the adsorbate fully equilibrates with the thermal bath presented by the lattice atoms. There are still some discrepancies present at low and high energies. We can conclude that at such low temperatures the bounce number $n_{b}=40$ is not sufficient to reach a quasi-stationary distribution close to the Boltzmann prediction, even though the trapped states dominate the DF for both angles already at $t>4.5 t_{0}(30 \mathrm{ps})$ according to the analysis presented in paper I 30 .

When comparing the results shown in figure 8 with the cases at higher temperatures displayed in figure 7. we actually observe that the latter DFs have converged to the Boltzmann curve specified by some effective temperature $T^{\star}$ for all three angles and $n_{b}=40$. In the case of a lattice temperature $T_{s}=190 \mathrm{~K}$ (left panel of figure 7), we obtain the value $T^{\star} \sim 150 \mathrm{~K}$. At $T_{s}=300 \mathrm{~K}$ the effect of the subthermal distribution becomes larger, and we find $T^{\star} \sim 200 \mathrm{~K}$. Compared to the equilibrium Boltzmann prediction of $T^{\star}=T_{s}$, the measured DF shows an increased population of low-energy states. All this indicates that while the full convergence to an equilibrium function has been reached, the effective adsorbate temperature remains different from the lattice temperature with $T^{\star}<T_{s}$. We note that a similar "lag" between $T^{\star}$ and $T_{s}$ has been measured for Ar on $2 \mathrm{H}-\mathrm{W}(100)$ in 39$]$ and for $\mathrm{Ar}$ on $\mathrm{Pt}(111)$ in 40 . Our fitted values of $T^{\star}$ are found to be in very good agreement with these experimental data.

On the quantitative level, the convergence to equilibrium can be defined in terms of the effective temperature of the adsorbate. By analyzing the normal and tangential components (figures 6, 7, and 8), we found that in most cases the DFs for $n_{b} \geq 9$ can be well fitted by the Boltzmann distribution

$P\left(E^{(n)}, n_{b}\right) \approx \frac{\pi}{T} \exp \left[-E_{k}^{(n)} / T^{(n)}\left(n_{b}\right)\right]$ 

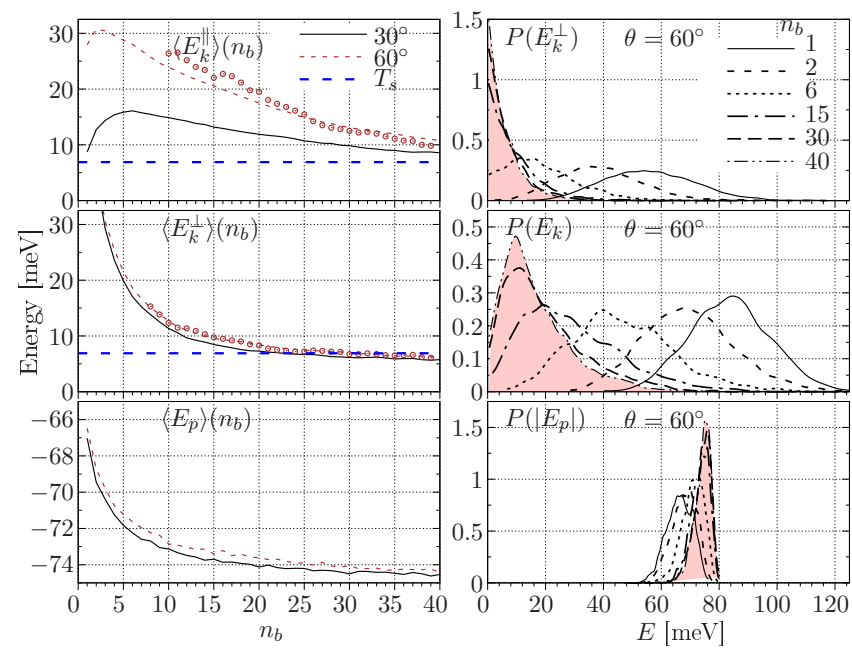

Figure 9. Left: Convergence of the parallel and normal components of the kinetic energy $\left(E_{k}^{\|}, E_{k}^{\perp}\right)$ and potential energy $\left(E_{p}\right)$ versus $n_{b}$, for the incident angles $\theta=30^{\circ}, 60^{\circ}$ and lattice temperature $T_{s}=80 \mathrm{~K}$. Right: Associated evolution of the distribution functions of $E_{k}^{\perp}$, full kinetic energy $\left(E_{k}\right)$ and $\left|E_{p}\right|$.
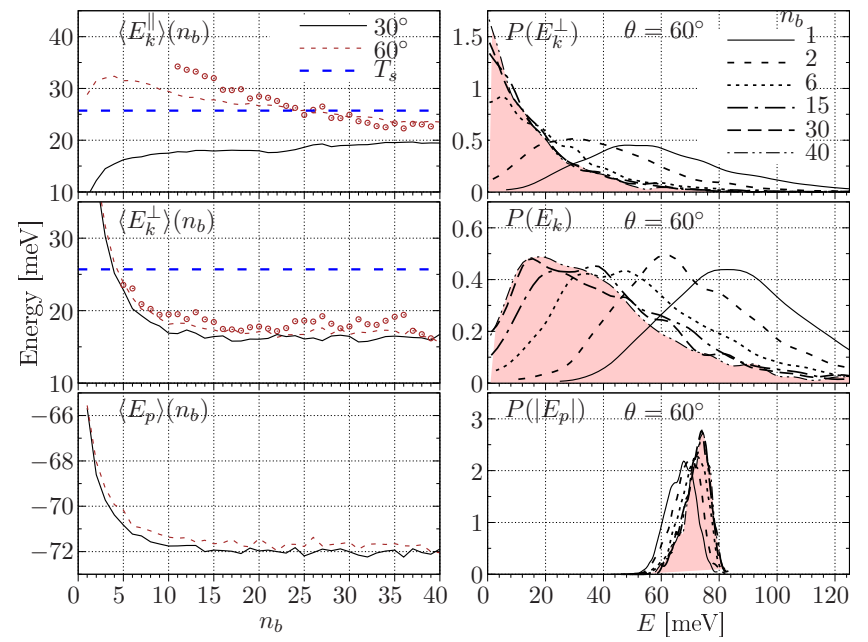

Figure 10. Same as in figure 9 , but for the lattice temperature $T_{s}=300 \mathrm{~K}$.

with the temperature $T$ used as a time-dependent fit parameter and $n=(\perp, \|)$. Using this relation, the average kinetic energy $\left\langle E_{k}^{(n)}\right\rangle\left(n_{b}\right)$, given by

$\left\langle E_{k}^{(n)}\right\rangle\left(n_{b}\right)=\int \mathrm{d} E_{k}^{(n)} E_{k}^{(n)} P\left(E_{k}^{(n)}, n_{b}\right)=T^{(n)}\left(n_{b}\right)$,

should coincide with a true kinetic energy component as soon as the model distribution (2) is close to the energy distribution determined by the MD simulations. Such an analysis is presented for the lattice temperatures $T_{s}=80 \mathrm{~K}$ and $300 \mathrm{~K}$ in figures 9 and 10, respectively. The two dashed lines in the two upper figures in the left panels show the decay of the MD kinetic energy versus the number of bounces. The open dots are the estimates based on Eq. (3), where the effective temperature $T^{(n)}$ has been introduced as a fit parameter [shown are the results for $\theta=60^{\circ}$.

Note that in the Boltzmann case the average kinetic energy is a measure of the temperature: $\left\langle E_{k}^{(n)}\right\rangle\left(n_{b}\right)=T^{(n)}\left(n_{b}\right)$. Hence, both dependences demonstrate how fast the adsorbate effective temperature converges. As shown in figure 9 for $T_{s}=80 \mathrm{~K}$, the temperatures $T^{\|}$and $T^{\perp}$ (and the kinetic energies $\left\langle E_{k}^{\|}\right\rangle$and $\left\langle E_{k}^{\perp}\right\rangle$ as well) approach some effective temperature $T^{\star}$. It is comparable with the lattice temperature $T_{s}$, which is shown by the horizontal dashed line. In agreement with our previous discussion, we observe a much faster relaxation of the normal component. For comparison, the convergence of the normal energy distribution, $P\left(E_{k}^{\perp}\right)$, and of the total kinetic energy distribution, $P\left(E_{k}\right)$, versus $n_{b}$ can be followed in the right panels. The two lower figures illustrate the behavior of the average potential energy $\left\langle E_{p}\right\rangle\left(n_{b}\right)$ as a function of bounce number and of the corresponding distribution function $P\left(\left|E_{p}\right|\right)$ for selected $1 \leq n_{b} \leq 40$.

The corresponding analysis for the higher lattice temperature, $T_{s}=300 \mathrm{~K}$, is presented in figure 10 . The main difference to the case with $T_{s}=80 \mathrm{~K}$ is that the converged effective temperature is $T^{\star}=200 \mathrm{~K}$ and is well below $T_{s}$.

The presented results bring us to the following conclusions:

i. The Boltzmann distribution can approximately fit the simulation data for the distribution functions.

ii. In this case, the average kinetic energy is a measure of the effective adsorbate temperature $T^{(n)}$.

iii. In the long term, the value $T^{(n)}$ approaches some stationary value, typically, below the lattice temperature $T_{s}$.

iv. The MD simulations allow us to identify the equilibration times for different incidence conditions $\left\{E_{i}, \theta, T_{s}\right\}$.

\section{Sticking coefficient}

Now we focus on evaluation of the sticking coefficient and its dependence on the lattice temperature and the incidence conditions. Using our subdivision of particle trajectories, it is clear that both trapped and quasi-trapped particles contribute to this macroscopic observable defined as

$R_{s t}\left(E_{i}, \theta, t\right)=N_{T}\left(E_{i}, \theta, t\right)+N_{Q}\left(E_{i}, \theta, t\right)$.

The contributions of the trapped and quasitrapped states to the initial sticking coefficient after the first bounce, $n_{b}=1$, corresponding to $t \lesssim 0.4 t_{0}$ $\approx 2.6 \mathrm{ps}$ are given in Table 2. In particular, it is found that the contribution of trapped particles to $R_{s t}$ decreases with increasing incident angle (and energy), while that of quasi-trapped particles increases at the same time. 
Table 2. Initial sticking probability and contributions of trapped $\left(N_{T}\right)$ and quasi-trapped $\left(N_{Q}\right)$ particles, cf. Eq. (4). Four incident angles and three lattice temperatures, $T_{s}$, are compared. The incident energies (in units of room temperature $\left.T_{r}\right)$ are $E_{i} /\left(k_{B} T_{r} / e_{0}\right)=0.5\left(0^{\circ}\right) ; 0.62\left(30^{\circ}\right) ; 0.84\left(45^{\circ}\right) ; 1.41\left(60^{\circ}\right)$.

\begin{tabular}{cccccc}
\hline$T_{s}$ & $\theta$ & $0^{\circ}$ & $30^{\circ}$ & $45^{\circ}$ & $60^{\circ}$ \\
\hline \multirow{3}{*}{$80 \mathrm{~K}$} & $N_{T}$ & 0.694 & 0.594 & 0.398 & 0.121 \\
& $N_{Q}$ & 0.095 & 0.173 & 0.317 & 0.594 \\
& $R_{s t}$ & 0.789 & 0.767 & 0.715 & 0.715 \\
\hline \multirow{3}{*}{$190 \mathrm{~K}$} & $N_{T}$ & 0.570 & 0.522 & 0.405 & 0.187 \\
& $N_{Q}$ & 0.094 & 0.154 & 0.231 & 0.466 \\
& $R_{s t}$ & 0.664 & 0.676 & 0.636 & 0.653 \\
\hline \multirow{3}{*}{$300 \mathrm{~K}$} & $N_{T}$ & 0.531 & 0.493 & 0.417 & 0.218 \\
& $N_{Q}$ & 0.074 & 0.124 & 0.194 & 0.402 \\
& $R_{s t}$ & 0.605 & 0.617 & 0.611 & 0.620 \\
\hline
\end{tabular}

The temperature dependence of the initial sticking coefficient for normal incidence $\left(\theta=0^{\circ}\right)$ can be compared with MD simulation results of Head-Gordon et al 33. Using the condition $E_{k}^{\perp}+E_{p}<0$ as the trapping criterion, they report $R_{s t}(80 \mathrm{~K})=$ $0.72, R_{s t}(180 \mathrm{~K})=0.62$ and $R_{s t}(300 \mathrm{~K})=0.58$. Our values are systematically larger by $3 \ldots 10 \%$. This discrepancy most probably originates from the different model used for the Pt-Pt and Ar-Pt interaction potentials. In Ref. 33 the platinum surface was described by a harmonic nearest neighbor and next-nearest-neighbor interaction, and the Ar$\mathrm{Pt}$ interaction by a 6-12 Lennard-Jones potential. Furthermore, the normal incident energy in Ref. 33. was $E_{i}=20.72 \mathrm{meV}$ compared to $E_{i}=12.85 \mathrm{meV}$ used in table 2 ,

\subsection{Energy-resolved initial sticking coefficient $\left(n_{b}=1\right)$. Comparison with experimental data}

During a collision event, the parallel momentum for a flat surface is conserved and does not play an important role in the trapping. Therefore, it can be assumed that the sticking probability depends solely on the energy exchange of the normal component in this ideal case, and that it scales as $R_{s t}\left(E_{i}, \theta\right) \sim R_{s t}\left(E_{k}^{\perp}\right)$ with $E_{k}^{\perp}=$ $E_{i} \cos ^{2} \theta$. If this assumption is valid, the measured sticking probabilities, obtained for a series of incident particle trajectories with the varying grazing angle $\theta$ and energy $E_{i}$, fit well with a common curve, when plotted as a function of $R_{s t}\left(E_{k}^{\perp}\right)$.

Possible deviations from this "normal scaling" $\left(\cos ^{2} \theta\right)$ imply that the scattering surface is corrugated, e.g. due to the binary gas-surface atom interaction. The scaling $R_{s t}\left(E_{i} \cos ^{n} \theta\right)$ with $n<2$ indicates a dependence on the parallel momentum as well, whereas the limit $n=0$ refers to the dependence of $R_{s t}$ on the total energy exchange between an atom and the surface.

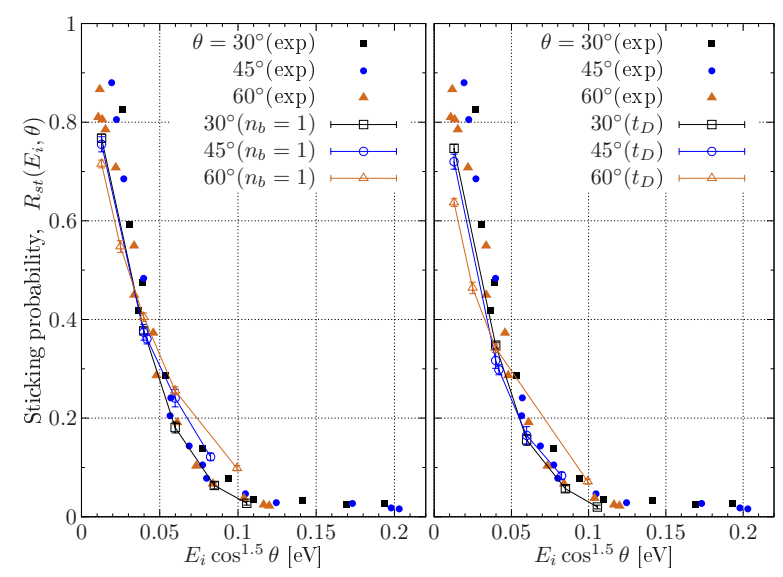

Figure 11. Dependence of the sticking coefficient $R_{s t}\left(E_{i}, \theta\right)$ for $\mathrm{Ar}$ on $\mathrm{Pt}(111)$ on the incident angle and energy for a lattice temperature $T_{s}=80 \mathrm{~K} . E_{i}$ is rescaled with $\cos ^{1.5} \theta$. Full symbols: our simulations. Open symbols: experimental data from Ref. 31]. Left: initial sticking coefficient after the first bounce $\left(n_{b}=1\right)$. Right: sticking coefficient after a delay time $t_{D}=10 \mathrm{ps}$ (see text).

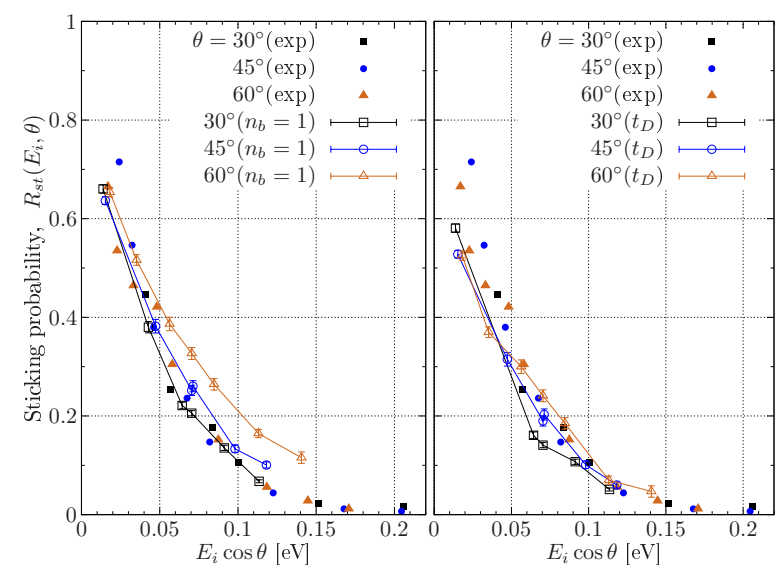

Figure 12. Same as Fig. 11 but for $T_{s}=190 \mathrm{~K}$ and a different energy scaling according to $E_{i} \cos \theta$. The sticking probability measured with the delay time $t_{D}=10 \mathrm{ps}$ (right panel) better agrees with the experiment 31 .

Energy-resolved initial sticking coefficients obtained by our MD simulations at the lattice temperatures of 80,190 and $300 \mathrm{~K}$ are shown in figures 11 . 12 and 13 , respectively. In our simulations the sticking coefficients have been evaluated at three angles, $\theta=\left\{30^{\circ}, 45^{\circ}, 60^{\circ}\right\}$, as a function of the incident energy $E_{i}$. The incident atoms were initially randomly distributed within the area of the simulation cell to provide an average over the scattering events covering the whole area of the simulated sample. The simulation results are compared with experimental data reported in 31 .

The sticking probability decreases with the incident energy $E_{i}$ as well as with the rescaled energy 
$E_{i} \cos ^{n} \theta$. Following the experimental data of Ref. 31, we used the exponents $n=1.5,1.0$ and 0.5 for the temperatures $T_{s}=80 \mathrm{~K}, 190 \mathrm{~K}$, and $300 \mathrm{~K}$, respectively. As a result of these scalings, most data points after the first bounce $\left(n_{b}=1\right)$ shown on the left side of figures 11, 12, and 13 nearly fall on a single curve for different combinations of $E_{i}$ and $\theta$.

Furthermore, much better agreement with the MD simulation results reported in 33] for $\theta=0^{\circ}$ can be resolved by the comparison with $R_{s t}\left(E_{i}, \theta\right)$ reported in figures 11, 12, and 13 at the rescaled energy $E_{i} \cos ^{n} \theta \sim$ $20 \mathrm{meV}$. Due to the expected universal dependence of $R_{s t}$, the results reported for $\theta=30^{\circ}$ and $45^{\circ}$ should agree with the ones of Ref. 33] for $\theta=0^{\circ}$. This comparison shows good coincidence of the results and demonstrates that both models provide a reasonable description of the gas-surface interaction energies and sticking in the thermal regime.

However, there still is a systematic discrepancy between our simulation results for the initial sticking coefficient after the first bounce and the experimental results, in particular, for the highest temperature (left side of figure 13). The origin of these deviations is analyzed in the following section, and a solution of this problem is presented.

\subsection{Energy-resolved stationary sticking coefficient. Choice of the delay time $t_{D}$}

The best agreement with the experimental data and the assumed scaling is observed at $T_{s}=80 \mathrm{~K}$ (figure 11). As it is shown in figure 3 of paper I [30, the scattered and the trapped states converge already after $t \sim 2 t_{0}-3 t_{0}$ corresponding to $13 \ldots 19$ ps. The defined trapped fraction is preserved on a long time scale according to the estimated quasi-equilibrium desorption rates $T_{C T}$ and $T_{C Q}$ (see figure 9 in paper I). Hence, there is less ambiguity in the definition of the trapped states both experimentally and in the simulations.

An uncertainty arises at the higher temperatures $T_{s}=190 \mathrm{~K}$ and $T_{s}=300 \mathrm{~K}$ (left sides of figures 12 and 13 . Once the desorption rates $T_{C T}$ and $T_{C Q}$ have finite values, the number of trapped states steadily decreases on a time scale of several picoseconds, $t \sim$ $15 \mathrm{ps}$ (see figures 5 and 7 in paper I). Hence, the sticking fraction is dependent on a delay time $t_{D}$ when the trapped particles can be desorbed at $t \leq t_{D}$ and counted as scattered. It is difficult to specify this parameter in the experiment. Thus, the question arises how to compare experiment and theory (simulation), and for what specific value of $t_{D}$ ? In the following, we discuss this problem more in detail.

First, we note that the assumed scaling $E_{i}(\theta)=$ $E_{i} \cos ^{n} \theta$ with the value $n$ used to fit the experimental data does not hold correctly for the MD data after

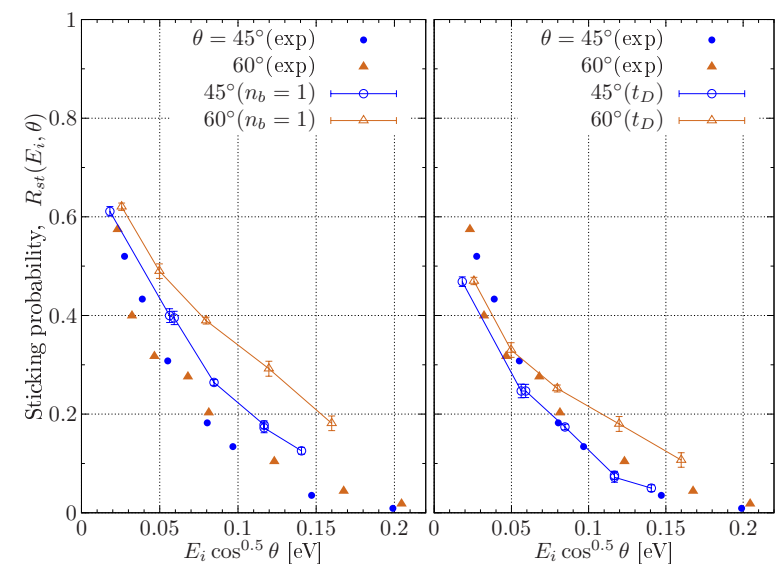

Figure 13. Same as in Fig. 12 but for $T_{s}=300 \mathrm{~K}$. The incident energy is rescaled as $E_{i} \cos ^{0.5} \theta$.

one bounce for higher temperatures at large energies and incident angles. Similar to the low temperature case $\left(T_{s}=80 \mathrm{~K}\right)$, we get quantitative agreement with the experimental results for $\theta=30^{\circ}$. However, deviations appear systematically for $\theta=45^{\circ}$ and $60^{\circ}$. They increase at larger energies, i.e., for $E_{i}(\theta)>$ $0.05 \mathrm{eV}$ in figure $12\left(T_{s}=190 \mathrm{~K}\right)$ and for $E_{i}(\theta)>$ $0.025 \mathrm{eV}$ in figure $13\left(T_{s}=300 \mathrm{~K}\right)$. The MD results (left panel) correspond to the initial trapped fraction and, hence, they should provide an upper bound for the trapped fraction measured with some time delay (experimental data). This is indeed the case for both the temperatures, and the difference between the predicted upper bound and the experimental data increases with $\theta$ and $E_{i}(\theta)$. In particular, the deviations at a fixed value of $E_{i}(\theta)$ are always larger for $\theta=60^{\circ}$ than for $\theta=45^{\circ}$. Note that the absolute energy $E_{i}$ is larger for $\theta=60^{\circ}$ due to the scaling factor $\cos ^{n} \theta(n \geq 1)$. If we now refer to the desorption rate $T_{Q T}(T, E)$ (see figure 9 in paper I), which corresponds to the first stage in the desorption process $\mathrm{T} \rightarrow \mathrm{Q} \rightarrow \mathrm{C}$, we find that its value increases with energy. Hence, the desorption fraction accumulated during a fixed time delay becomes larger for $\theta=60^{\circ}$ than for $\theta=45^{\circ}$. In our opinion, this is the main reason for the increase of deviations at larger $\theta$ and $E_{i}(\theta)$.

In order to make a proper comparison, we propose to introduce a fit parameter $t_{D}$, i.e., a delay time specific to a given experiment and related to a setup used to identify the desorbed (or the trapped) fraction. The sticking probability defined in the experiment can be written as

$$
\begin{aligned}
& R_{s t}^{\exp }\left(E_{i}, \theta\right) \approx R_{s t}^{\mathrm{MD}}\left(E_{i}, \theta, t_{D}\right)=1-N_{C}\left(t_{D}\right), \\
& N_{C}\left(t_{D}\right)=N_{C}\left(t_{1}\right)+\mathrm{d} N_{C}\left(t_{1}, t_{D}\right), \\
& \mathrm{d} N_{C}\left(t_{1}, t_{D}\right)=\int_{t_{1}}^{t_{D}} \mathrm{~d} t\left[T_{C Q}(t) N_{Q}(t)\right.
\end{aligned}
$$




$$
\left.+T_{C T}(t) N_{T}(t)\right],
$$

where $N_{C}\left(t_{1}\right)$ is the fraction of atoms which experience a direct inelastic scattering to continuum by the first reflection from the surface (at some average time $t_{1}$ ), $\mathrm{d} N_{C}\left(t_{1}, t_{D}\right)$ is the contribution from the atoms, which have been initially trapped but desorbed during the time interval $t_{1}<t \leq t_{D}$. Equation (7) specifies the relation to the transition rates from the quasi-trapped and trapped states to the continuum.

For the present analysis, we defined $t_{D} \sim 1.5 t_{0} \sim$ $10 \mathrm{ps}$ empirically from a fit of a single MD data point to a single experimental point, taken at the specific lattice temperature, $T_{s}=190 \mathrm{~K}$, the incident angle, $\theta=45^{\circ}$, and the energy, $E_{i}(\theta) \sim 0.12 \mathrm{eV}$, i.e.,

$R_{s t}^{\exp }\left(E_{i}, \theta, T_{s}\right)=R_{s t}^{\mathrm{MD}}\left(E_{i}, \theta, t_{D}, T_{s}\right)$.

The sticking probability, defined via Eq. (5) with the fixed delay time $t_{D}=1.5 t_{0}$, is now compared with the experimental data. This comparison is displayed in the right panels of figures 11, 12, and 13, In general, we observe much better agreement taking into account that the value $t_{D}$ was not adjusted separately in each case. The results for $T_{s}=80 \mathrm{~K}$ remain practically unchanged, as there is no noticeable thermal desorption on the time scale $t \leq t_{D}$. Much better agreement is now observed for $\theta=45^{\circ}$ and $60^{\circ}$ at $T_{s}=190 \mathrm{~K}$ (figure 12 ) and for $\theta=45^{\circ}$ at $T_{s}=300 \mathrm{~K}$ (figure 13). The remaining discrepancy for $\theta=60^{\circ}$ and $T_{s}=300 \mathrm{~K}$ can be further reduced by choosing $t_{D} \sim(2 \ldots 3) t_{0}$.

Now we argue that a proper choice of $t_{D}$ should be the quasi-equilibration time $t^{E}$. This is related with the procedure, how the trapped fraction is determined experimentally. Typically, the procedure is well defined at low lattice temperatures $(\sim 80 \mathrm{~K})$. The detected kinetic energy distribution in the angleresolved reflected flux shows a bimodal distribution with one sharp and a second broad maxima. The first maximum is related with the specular reflection (corresponding to one-two bounces with the surface), and its position shows a strong correlation with the incidence conditions. In contrast, the second maximum stays independent, and, therefore, is related with the trapping-desorption contribution of particles which are temporarily trapped near the surface and partially equilibrate. The relative contribution of both varies strongly with the detection angle 22 , 41, 42. By choosing the detection angle far from the incident one (where the specular reflection dominates), the measured intensity is mainly due to the trapped and quasi-trapped states. By analyzing the temporal evolution of the energy spectra related with the trapping-desorption fraction, one can study its convergence to a quasi-stationary form, and extract the quasi-equilibration time $t^{E}$. Then, the integrated intensity taken at $t \geq t^{E}\left(=t^{D}\right)$ can be used as a definition of the trapped fraction and the sticking coefficient. The resolved characteristic time $t^{E}$ can be compared versus the MD simulations. From the theoretical side, the value of $t^{E}$ can be determined independently using several of the criteria mentioned in the previous sections. This includes the convergence of the kinetic energy distributions, the convergence of the average kinetic energy, and the time-dependence of the transition rates, cf. paper I [30].

\section{Conclusion and outlook for plasma-surface interaction}

One of the fundamental aspects of rare gas-metal surface scattering experimental studies is the phenomenological division 42 44] of this process into two distinct channels: the direct inelastic and the trapping desorption one. At shorter times a beam energy-dependent peak is observed that is attributed to direct inelastic scattering. On the other hand, at longer time scales, the energy (time-of-flight) spectra exhibit a second, beam energy-independent peak that is attributed to the trapping desorption scattering. It shows nearly Maxwellian behavior at the surface temperature $T_{s}$. These experimental findings get a firm conformation from the present MD simulations, where the adsorbate equilibration kinetics has been studied in detail. By performing relatively long simulations we are able to distinguish and quantify two characteristic regimes. At shorter times, i.e., within the first $5-10$ bounces with the surface, corresponding to a few picoseconds, the energy distribution functions of the adsorbate atoms exhibit fast changes. Then, at later times they converge to a quasi-stationary form, which stays practically unchanged until the end of the simulations. This fact confirms that the phenomenological assumption of the two types of scattering processes is indeed valid. However, we also found that the corresponding time scales, for which these processes can be well separated, strongly depend on the incidence conditions of the atoms and on the lattice temperature. It was one of the goals of the present study to make quantitative predictions on such relevant time scales. The results can be used for a better interpretation of experimental energy-resolved (time-of-flight) spectra.

The combination of the present MD simulations, based on microscopic gas-surface and binary atomatom interactions, with the rate equation model, described in more detail in paper I [30], allows us to directly analyze the temporal evolution of the trapping-desorption fraction. This quantity is difficult to access experimentally. Therefore, our combined model allows for valuable predictions of the time scales when the behavior of the trapping-desorption 
fraction changes from a non-stationary one to a quasiequilibrium state. This was demonstrated on the example of several macroscopic observables.

The temperature dependence of the ELDF has also been analyzed. The half-width of the ELDF exhibits a linear scaling with the lattice temperature $T_{s}$. In the quasi-equilibrium regime $\left(t>t^{E}\right)$ the ELDF is symmetric with respect to zero energy. This justifies that the probabilities of excitation and deexcitation become equal due to the energy exchange with the lattice. In this case the adsorbate average kinetic energy fully accommodates to some effective temperature $T^{\star}$ which is comparable to $T_{s}$. In general, we found that the accommodation time increases with the population of the quasi-trapped states $N_{Q}$. The latter increases with the angle of incidence of the gas atoms.

The convergence of the parallel and normal components of the kinetic energy has been studied for several typical incident angles and $T_{s}$ values. In agreement with previous studies 32 34 45 , the parallel component always shows a slower convergence. The time-resolved kinetic energy distribution functions can be fitted by a Maxwellian distribution at some effective temperature, which typically shows a (subthermal) "lag" with respect to the lattice temperature.

Finally, we checked the quality of our model by comparison with experimental data of the sticking coefficient 31]. While the experimental results for low lattice temperature $\left(T_{s}=80 \mathrm{~K}\right)$ have been accurately reproduced, systematic discrepancies are observed for higher temperatures $\left(T_{s}=190 \mathrm{~K}\right.$ and $\left.300 \mathrm{~K}\right)$. To get better agreement with the experimental data, we proposed to introduce a delay time, $t_{D}$, which takes into account the fraction of atoms which have been initially trapped, but have desorbed at $t<t_{D}$ and counted in the experiment as scattered. The empirical choice of this fit parameter, as $t_{D} \sim t^{E}(10-30 \mathrm{ps})$, i.e., of the order of the quasi-equilibration time, brings our MD data into much better agreement with the experiments. This fact most likely explains similar discrepancies that were observed in other numerical simulations as well, see Refs. 32, 34, 46

In summary, we studied the trapping-desorption processes, being the dominant mechanism for the scattering of rare gas atoms from metal surfaces, for the case of thermal and subthermal impact energy. The simulation results generally agree with the experimental data and provide estimates of the characteristic quasi-equilibration times, the energy loss and kinetic energy spectra. The effects of the lattice temperature and incidence conditions have been analyzed in detail. Our theory and simulations are expected to be a useful tool for the analysis of atomic and molecular scattering, when the trapping- desorption mechanism or the initial trapping fraction (relative to the direct-scattered fraction) dominate.

Our results are expected to be of particular interest for low-temperature plasmas at low pressure for which the considered energy range is of relevance. Due to the low degree of ionization the behavior of neutral gas atoms in the vicinity of a solid surface is crucial both, for fundamental understanding and for many applications, for a recent overview see Ref. [47. First of all, our results for the sticking probability can be used to compute improved sticking coefficients of rare gas atoms on a metal surface for typical low-temperature plasma conditions. To this end, the present results for monoenergetic atoms can be averaged with the proper energy and angle distribution of atoms. Moreover, the same procedure can be applied to nonequilibrium conditions in the plasma sheath which are resulting e.g. from charge-exchange collisions.

Second, our results could serve as an input for particle based simulations, such as particle-incell, molecular dynamics or kinetic Monte Carlo simulations, see Ref. [48 and references therein. The present energy and angle resolved sticking probabilities should allow for much more accurate simulation of surface processes that have predictive capability.

Third, the present method can be easily extended to other material combinations, in particular for metal surfaces, provided suitable ab initio pair potentials or force fields are available that can be used as input for MD simulations.

Fourth, the capability of our combined MDrate equation approach to perform accurate long-time simulations can be a valuable starting point to study slow processes such as surface modification due to sputtering or film growth. For example, our approach is not limited to an ideal metal surface but could be equally applied to a realistic corrugated surface that includes steps or defects.

Finally, our approach should, in principle, also allow for an $a b$ initio study of adsorption processes. Here, the main difference compared to the present study is that sticking of atoms is not independent of the state of the surface. Instead, it is possible to track the pre-existing adsorbed atoms and include their influence on the sticking of atoms that arrive at a later point in time. The present MD simulations can be easily extended by including proper atom-atom pair potentials. At the same time, the present rate equation model 30 can be straightforwardly extended to inhomogeneous systems and to the computation of atomic pair distribution or triple correlation functions 49. This provides a suitable route to the computation of structural properties of surfaces in low-temperature plasmas and to the comparison with 
experimental data.

\section{References}

[1] Hayes W W and Manson J R 2007 Phys. Rev. B 75, 113408; Hayes W W, Ambaye H and Manson J R 2007 J. Phys.: Condens. Matter 19,305007

[2] Pollak E and Miret-Artés S 2009 J. Chem. Phys. 130, 194710

[3] Gibson K D, Silbener S J, Upadhyaya H P et al $2008 \mathrm{~J}$. Chem. Phys. 128, 224708

[4] Farías D and Rieder K H 1998 Rep. Prog. Phys. 61, 1575

[5] Gumhalter R 2001 Phys. Rep. 351, 1

[6] Guantes R, Santz A S et al 2004 Surf. Sci. Rep. 53, 199; Santz A S and Miret-Artés S 2007 Phys. Rep. 451, 37

[7] Schweizer E K and Rettner C T 1989 Phys. Rev. Lett. 62, 3085; Schweizer E K, Rettner C T and Holloway S 1991 Surf. Sci. 249, 335

[8] Rieder K H and Stocker W 1985 Phys. Rev. B 31, 3392

[9] Berenbak B et al 2002 Chem. Phys. 4, 68

[10] Castejón H J 2004 Surf. Sci.. 564, 165

[11] Raukema A, Dirksen R J and Kleyn A W 1995 J. Chem. Phys. 103, 6217

[12] Rettner C T, Barker J A, and Bethune D S 1991 Phys. Rev. Lett. 67, 2183

[13] Barker J A, Rettner C T, and Bethune D S 1992 Chem. Phys. Lett. 188, 471

[14] Lahaye R J W E, S. Stolte, Kleyn A W, Smith R J, and Holloway S 1994 Surf. Sci.. 187, 307

[15] Brako R 1982 Surf. Sci. 123439

[16] Tully J C 1990 J. Chem. Phys. 92, 680

[17] Yan T, Hase L, and Tully J C 2004 J. Chem. Phys. 120, 1031

[18] Hubbard L M and Miller W H 1984 J. Chem. Phys. 80, 5827

[19] Pollak E 2011 J. Phys. Chem. A 115, 7189

[20] Zhou Y, Pollak E, and Miret-Arts S 2014 J. Chem. Phys. 140, 024709

[21] Sahoo T and Pollak E 2015 J. Chem. Phys. 143, 064706

[22] Fan G and Manson J R 2009 Phys. Rev. B 79, 045424

[23] Medina Z and Jackson B 2008 J. Chem. Phys. 128, 114704

[24] Lepetit B, Lemoine D, Medina Z and Jackson B $2011 \mathrm{~J}$ Chem. Phys. 134, 114705

[25] Bonfanti $\mathrm{M}$ et al $2015 \mathrm{~J}$. Chem. Phys. 143, 124703; M. Bonfanti et al $2015 \mathrm{~J}$. Chem. Phys. 143, 124704

[26] Castejón H and Bernecker C 2012 Surf. Sci. 606, 1274

[27] Iqbal S A, Mido Y 2005 Physical chemistry, Discovery Publishing House

[28] D.A. McQuarrie 2008 Quantum chemistry, University Science Books

[29] Sun J and Li Z-X 2011 Heat Transfer Eng. 32, 658

[30] Filinov A, Bonitz M, and Loffhagen D, preceding paper (paper I), arXiv:1802.03466

[31] Mullins C B, Rettner C T, Auerbach D J, and Weinberg W H 1989 Chem. Phys. Lett. 163, 111

[32] Smith R J, Kara A and Holloway S 1991 J. Chem. Phys. 94, 806

[33] Head-Gordon M, Tully J C, Rettner C T, Mullins C B and Auerbach D J 1991 J. Chem. Phys. 94, 1516; HeadGordon M and Tully J C 1992 Surf. Sci. 268, 113

[34] Smith R J, Kara A and Holloway S 1993 Surface Science 281, 296

[35] Tully J C 1981 Surf. Sci.. 111, 461

[36] Tully J C 1994 Surf. Sci.. 299, 667

[37] Spijker P, Markvoort A J, Nedea S V and Hilbers P A J 2010 Phys. Rev. E 81, 011203

[38] Léonard C, Brites V, Pham T T, To Q D, and Lauriat G 2013 Eur. Phys. J. B 86, 164
[39] Rettner C T, Schweizer E K, and Mullins C B 1989 J. Chem. Phys. 90, 3800

[40] Hurst J E, Wharton L, Janda K C, and Auerbach D J 1985 J. Chem. Phys. 83, 1376

[41] Chirita V, Pailthorpe B A and Collins R E 1993 J. Phys. D: Appl. Phys. 26, 133

[42] Janda K C, Hurst J E, Becker C A, Cowin J P, Auerbach D J and Wharton L 1980 J. Chem. Phys. 72 (4), 2403

[43] Janda K C, Hurst J E, Becker C A, Cowin J P, Auerbach D J, and Wharton L 1980 Surf. Sci.. 93, 270

[44] Hurst J E, Janda K C, Becker C A, Cowin J P, Auerbach D J and Wharton L 1979 Phys. Rev. Lett. 43, 1175

[45] Hurst J E, Wharton L, Janda K C and Auerbach D J 1983 J. Chem. Phys. 78, 1559

[46] Kulginov D, Persson M, Rettner C T and Bethune D S 1996 J. Phys. Chem. 100, 7919

[47] Adamovich I, Baalrud S D, Bogaerts A, Bruggeman P J, Cappelli M, Colombo V, Czarnetzki U, Ebert U, Eden J G, Favia P et al 2017 J. Phys. D: Applied Physics 50, 323001

[48] Bonitz M, Rosenthal L, Fujioka K, Zaporojtchenko V, Faupel F and Kersten H 2012 Contrib. Plasma Phys. 52 (10), 890-898

[49] Thomsen H and Bonitz M 2015 Phys. Rev. E 91043104 